\title{
Si può distinguere utopistico da utopico oppure... è un'utopia?
}

\author{
Paolo D'Achille
}

PUBBLICATO: 09 OCTOBER 2020

\section{Quesito:}

Ci sono arrivati vari quesiti che chiedono se tra utopistico e utopico vi sia una differenza di significato oppure no; se utopico si possa considerare corretto o quanto meno se sia corretto il suo riferimento a idee (idea utopica o utopistica?) o a persone (invece di utopista); se ci sia un legame semantico tra utopico e idilliaco.

Si può distinguere utopistico da utopico oppure... è un'utopia?

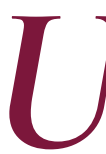

Topico, utopista e utopistico sono tutti e tre derivati da Utopia, nome dell'isola immaginaria in cui è ambientata unopera del filosofo inglese Thomas More (in italiano Tommaso Moro), scritta in latino (Libellus vere aureus, nec minus salutaris quam festivus de optimo rei publicae statu deque nova Insula Utopia) e pubblicata nel I516. Il nome sembra anticipare quello di Neverland 'I'Isola che non c'e' di Peter Pan, forse non a caso "inventato" da un altro autore britannico, lo scozzese James Matthew Barrie all'inizio del Novecento.

Il nome di Utopia si potrebbe definire come uno pseudo-grecismo, in quanto non esisteva nel greco antico, anche se è formato con due parole greche, l'avverbio ou 'non' e il nome tópos 'luogo', con l'aggiunta del suffisso -ia usato per formare appunto nomi di luogo sia in greco sia anche in latino (ma con l'accento sulla sillaba precedente, come andrebbe fatto leggendo il titolo latino dell'opera). A Utopia si collegano gli aggettivi utópio e utopiense, registrati nel GDLI e nel GRADIT, documentati nella traduzione italiana effettuata da Ortensio Lando nel 548 (La Repubblica del governo dell'Isola Utopia) e modellati su quelli (in latino moderno) del testo di Moro, Utopius e Utopienses. Questi due aggettivi (riprodotti senza modifiche nella riedizione della traduzione cinquecentesca stampata a Milano, da Vincenzo Ferrario, nel I848 con la premessa di Pietro Giordani) significano 'dell'isola immaginaria di Utopia' e, come etnici (e dunque anche in funzione di nomi), "che, chi abita nell'isola di Utopia" (utopio, invero, nelle due traduzioni citate è usato un'unica volta, al plurale e con l'iniziale maiuscola, solo come nome). Ad essi nel Settecento si è aggiunto, con lo stesso significato, anche il derivato utopiano, per probabile influsso del francese utopien e dell'inglese utopian, a loro volta formati sull'Utopianus del testo latino di Moro, che in queste lingue significano anche 'utopico', 'utopistico'.

Fin dai primi del Seicento in italiano il nome dellisola di Tommaso Moro è stato usato in senso antonomastico come nome comune, prima nel significato di 'ideale irrealizzabile, progetto che non può avere un'attuazione pratica', poi, nell'ambito filosofico e politico, in quello di 'modello politico, sociale o religioso che non trova effettivo riscontro nella realtà ma che viene proposto come ideale' (entrambe queste definizioni, come pure quelle successive, sono tratte dal GRADIT).

Proprio con riferimento alla seconda e più specifica accezione, all'inizio dell'Ottocento in scritti filosofico-politici si diffusero nuovi termini legati a utopia: l'aggettivo utopico 'che ha i caratteri dell'utopia'; il nome (usato anche come aggettivo) utopista 'sostenitore, seguace di unutopia' e poi anche, in generale, 'chi ha ideali e progetti irrealizzabili'; l'aggettivo utopistico 'proprio di un'utopia o degli utopisti' e 'che ha le caratteristiche di unutopia, irrealizzabile' Le date indicate nel GRADIT sono I897 per utopico (dall'epistolario di Antonio Labriola), I823 per utopista e I838 per utopistico e 
farebbero pensare che utopico sia più recente (il che spiegherebbe la "censura" scolastica segnalata da un lettore, che potrebbe affondare le radici nel purismo tardo-ottocentesco). Ma lo Zingarelli anticipa questa data al i 837 , riavvicinando cosi questo derivato agli altri due.

La ricerca in Google libri consente di cogliere, per due dei tre termini, qualche attestazione ancora anteriore. Per utopico risaliamo al I823 (ma riferito a romanzo: il senso è dunque quello di 'simile all'Utopia', con esplicito o implicito rimando al testo di Tommaso Moro):

VI Storia del primo stabilimento delle leggi, ec. de' Cessares, popolo dell'America meridionale, specie di romanzo utopico con forma di lettere, in 8.vo, 1760 . (s.v. Burgh [Giacomo], in Biografia universale antica e moderna, vol. VIII, Venezia, Missiaglia, I823, pp. 346-347, a p. 347)

[...]; queste quattro parti della filosofia sono la fisica, la morale, l'economia e la politica; è di quest'ultima la Cittá del sole, maniera di romanzo utopico, cui lo stesso Campanella qualificava di superiore molto alla Repubblica di Platone, ma che fu da Corringio, e con ragione, detto inferiore a quello di Tommaso Moro. (s.v. Campanella (Tommaso), ivi, vol. IX, I823, pp. 204-208, a p. 207)

Di utopistico anticipiamo l'apparizione al I829:

E so, che a molti, il vocabolo di Letteratura Europea suona distruzione d'ogni spirito nazionale d'ogni carattere individuale de' popoli; ad altri, stranezza, sogno utopistico. (D'una letteratura europea, in "Antologia", XXXVI, I829, pp. 9I-I00, a p. 93)

Utopista - probabilmente formato sulla scia del francese utopiste, che il TLFi data al 1792 - non è al momento retrodatabile, perché l'esempio più antico (nel periodico "La voce della verità. Gazzetta dell'Italia centrale", I33, I2 giugno I832, in un articolo che propone il "DIALOGO tra un Utopista, un Liberale sincero, ed un Realista") è posteriore al passo di Carlo Botta riportato nel GDLI, fonte per la datazione del GRADIT e dello Zingarelli.

Si può invece anticipare sempre al I832 utopismo, datato nel GRADIT I 899:

Questa dottrina Dantesca che traluce da tutta la Divina Commedia, è, a parer mio almeno, quella, che ha salvato il dominio temporale della Chiesa Romana negli ultimi tempi dell'utopismo politico, e della intraprendentissima ambizione Napoleonica. (Leonardo Antonio Forleo, Il manoscritto di Sterne ovvero Parte secunda del Viaggio sentimentale, Napoli, Cataneo, 1832, p. 357)

Questa datazione aumenterebbe l'anteriorità del termine italiano rispetto ai corrispondenti in francese (utopisme) e in inglese (utopism), datati nel TLFi rispettivamente igor e i888. In realtà, però, mentre resta posteriore l'inglese utopism, anche nel primo esempio citato nell'OED, del I849 (data confermata dalla ricerca su Google libri), in francese troviamo utopisme già in un testo del I830 (peraltro scritto da un italiano):

Pour ce qui est de mes doctrines politiques, je les abandonne également avec confiance au jugement du lecteur éclairé et impartial, persuadé que je suis qưil les trouvera, comme moi, aussi éloignées du servilisme que de l'utopisme libéral, ainsi appelé par certains disciples de l'école tremblottante. (J.B. [Giovanni Battista] Marochetti, Indépendance de l'Italie. Moyen de l'établir dans l'intérêt général de l'Europe, nouvelle édition, Paris, chez les Marchands de Nouveautés au Palais Royal, ı83o, p. XVII)

Tornando ai nostri utopico, utopista e utopistico, possiamo dire che tutti e tre (anzi, tutti e quattro con utopismo) sono sostanzialmente coevi e fin dai primi esempi hanno gli ambiti d'uso indicati nel GRADIT (e in altri dizionari): utopista si riferisce a persone, utopistico a cose, e cosi pure utopico. È 
dunque certamente improprio usare utopico con riferimento a persone, definendo utopico un politico, un filosofo, o magari un influencer, anche se è probabile che l'uso si stia diffondendo, favorito dal fatto che utopista è sentito piuttosto come nome e che utopistico non è utilizzabile in questa funzione, tanto più che, anche con riferimento a cose, gli aggettivi in -istico, pur se in grande espansione nel Novecento (Migliorini) da tempo hanno iniziato a cedere il campo alle forme in -ista (Fache). Infatti, come notato anche da Anna M. Thornton (in Grossmann-Rainer 2004, p. 527): "Luso aggettivale di nomi politico-ideologici in -ista nel Novecento è apparso in espansione, a danno degli aggettivi in istico; gli studiosi concordano nell'attribuire questa espansione a un influsso del francese".

Relativamente alla possibile differenziazione tra utopico e utopistico, i due aggettivi, formati entrambi con il suffisso -ico, in un caso aggiunto a utopia, nell'altro a utopista, hanno certamente un ampio margine di sovrapponibilità. Il Vocabolario Treccani online considera utopico "Forma più elevata e rara di utopistico"; si direbbe piuttosto che utopistico sia preferito con riferimento a idee, concezioni, programmi politici concreti, per quanto considerati irrealizzabili (ricordiamo il socialismo utopistico del primo Ottocento), mentre utopico abbia un significato un po' più astratto (pensiero utopico); ma si tratta di sfumature, che lasciano spazio alla soggettività.

Non trova riscontro nella lessicografia la differenza semantica proposta nel sito Storiologia.it, di cui ho tuttavia trovato qualche conferma da parte di persone amiche e che sembra documentare una tendenza dell'italiano a distinguere i significati dei tanti termini appartenenti alla stessa "famiglia lessicale" e almeno in parte equivalenti:

Ed è quindi bene operare una distinzione tra "utopico" ed "utopistico": un progetto utopico si distingue da uno utopistico per il fatto che è altamente positivo, degno di essere concretizzato; sotto questo profilo, l'abolizione della schiavitù può essere definita utopica, un modello "buono" da cui trarre ispirazione. Al contrario, qualora non sussistesse la schiavitù, sarebbe utopistico il progetto di ripristinarla: sarebbe cioè un qualcosa di negativo, indegno di essere applicato. (L'utopia-cos'é, 5/2/2003)

Quanto al rapporto semantico tra utopico e idilliaco, ipotizzato da una lettrice, una studentessa che fa riferimento alla lezione di un suo professore, il quale "ha usato prima l'aggettivo 'utopico' poi "idilliaco" (entrambi, probabilmente, a lei non proprio familiari), si può spiegare col fatto che questo secondo aggettivo significa anche "che deriva da un atteggiamento di ingenuo ottimismo nei confronti della realtà: avere una visione idilliaca della vita" (GRADIT); tale significato è avvicinabile semanticamente a quello di utopico; ma non si può certamente parlare di sinonimia.

Riportiamo ora i dati offerti da Ngram Viewer, grafico che rappresenta la distribuzione delle tre forme considerate nel corpus italiano di Google libri dal I80o al 2000 e oltre.

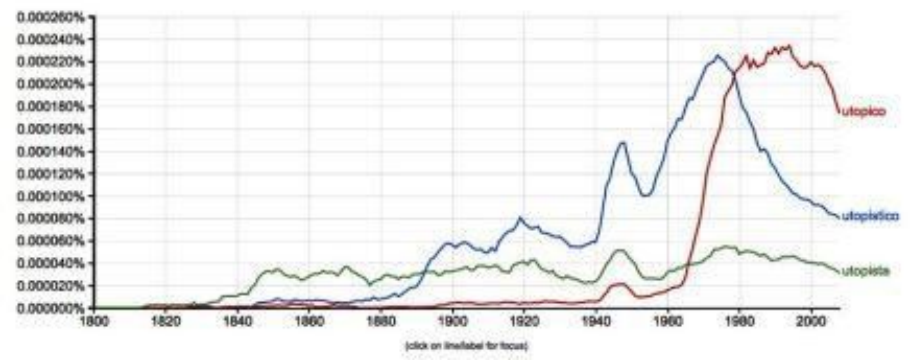

Il grafico mostra che utopista ha mantenuto un andamento sostanzialmente stabile ed è stato il termine più usato solo fino agli anni novanta del sec. XIX, quando è stato superato da utopistico, a sua volta in progressiva crescita fino al top degli anni Settanta, ma poi in costante flessione; quanto a utopico, rarissimo fino al 1940, ha poi avuto una progressiva espansione, superando utopista verso la 
fine degli anni Sessanta e utopistico nei primi anni Ottanta. Il grafico non consente di distinguere tra uso nominale e aggettivale di utopista, ma se ne può certamente dedurre che anche (e soprattutto) come aggettivo utopista sia molto meno frequente di utopistico e utopico.

Una conferma della tendenza evidenziata si coglie anche nel corpus di romanzi del PTLLIN, in cui troviamo un solo esempio di utopista come nome: "'austero e ascetico utopista" (Pietro Citati, Tolstoj, I984); tre occorrenze di utopistico/-i risalenti agli anni Cinquanta: "il gusto utopistico per le cose pratiche e precise" (Carlo Levi, L'orologio, I95I); "gli strumenti con cui fanno la guerra, tra primitivi puerili e utopistici" (Corrado Alvaro, Quasi una vita, I95I); "Tutto ciò [...] rendeva inverosimile, utopistico, un simile sogno" (Elsa Morante, Lisola di Arturo, 1957); cinque attestazioni di utopico/-a/-i negli anni Ottanta: "utopico futuro" (2 volte), "pensiero utopico" e "creazione utopica" (Claudio Magris, Damubio, I987); "disegni [...] utopici” (Stanislao Nievo, Le isole del paradiso, 1987).

L'affermazione del Vocabolario Treccani circa la maggiore rarità di utopico rispetto a utopistico vale dunque solamente con riferimento al passato ed è ben possibile che oggi utopico venga anche riferito a persone. Speriamo di non passare per utopisti se sconsigliamo quest'uso, indicandolo come improprio.

\section{Nota bibliografica:}

- Bruno Migliorini, Appunti sulla lingua contemporanea, II. Il suffisso -istico, in "Cultura neolatina", n.s., X (1931), pp. 976-984 (rist. in Bruno Migliorini, Saggi sulla lingua del Novecento, Firenze, Sansoni, I9633, pp. 99-I44).

- Charles Fache, L'aggettivazione dei nomi in -ista, in "Lingua nostra", XXXIV, 1973, pp. 84-88.

\section{Cita come:}

Paolo D'Achille, Si può distinguere utopistico da utopico oppure... è un'utopia?, "Italiano digitale", 2020, XV, 2020/4 (ottobre-dicembre)

DOI: $10.35948 / 2532-9006 / 2020.4408$

\section{Copyright 2020 Accademia della Crusca}

Pubblicato con licenza creative commons CC BY-NC-ND 